\title{
EL ÚLTIMO QUIPUCAMAYOC DE LA CULTURA WANKA, EL ECONOMISTA Y EL CONTADOR PÚBLICO*
}

\author{
Agüero Angulo, Martina ${ }^{1}$, y Álvarez Bernuy, Silvia Marina ${ }^{2}$ \\ Facultad de Economía de la Universidad Nacional del Centro del Perú
}

\begin{abstract}
RESUMEN
La ciencia económica es el resultado de un proceso largo en el mundo y en el Perú, sin embargo aún cuando no se definía, en el imperio incaico existió técnicas de registro estadístico y contable, incluso el Colegio de Economistas del Perú, utiliza como símbolo la imagen del Quipucamayoc y los Contadores Públicos usan el mismo símbolo, aunque no de modo oficial. El problema investigado: ¿existió el Quipucamayoc en la cultura Wanka en el proceso de colonización del Perú? ¿Quién fue el último Quipucamayoc de la cultura Wanka? Los objetivos fueron conocer los antecedentes históricos post inca de los Contadores Públicos y los Economistas en la cultura o etnia Wanka, el Quipu y el Quipucamayoc, de su manejo en el periodo de la conquista y colonización de Perú. El alcance de la investigación es a nivel regional e institucional, utilizando el método descriptivo y como muestra el $50 \%$ del personal docente ordinario de las Facultad de Contabilidad y de Economía. El periodo de la ejecución de la investigación ha sido de un año calendario. Las conclusiones importantes: los líderes Wankas, aprendieron a utilizar el quipu, se aliaron a los conquistadores entregándoles guerreros, cargadores, animales, bienes como alimentos, vestidos, herramientas, enseres, utensilios, etc., los mismos que fueron registrados en el quipu de cada Quipucamayoc. Los incas dividieron a los Wankas en 3 huamanis: Jatun Xauxa, Ananhuanca y Lurinhuanca y a la llegada de los españoles a Cajamarca, estos huamanis eran gobernados por Apo Manco Suchaqui, Apo Alaya Chuquillanqui y, Apo Manco Guacrapaucar, respectivamente quienes conocían el manejo de los quipus, considerándose al Apo Manco Guacrapaucar como el último Quipucamayoc de la cultura o etnia Wanka posteriormente bautizado por los españoles como Jerónimo Guacrapaucar, quien ha registrado en los quipus la cantidad de servicios y bienes entregados a los españoles para la conquista y colonización del Perú. Además existe similitud de algunas funciones ejecutadas por el Quipucamayoc con las del Contador Público y del Economista.
\end{abstract}

Palabras clave: estadístico, contable, huamanis, quipu, yupana

\section{THE LAST KUPUCAMAYOC IN THE WANKA CULTURE, THE ECONOMIST AND PUBLIC ACCOUNTANT UNCP FACULTY OF ECONOMY}

\begin{abstract}
The economic science is the result of a long proces in the world as well as in Peru howerer ever though it wasn't defined clearly in the Incas Empire existed recording coun table techiques, whats mose, the profesional school of economists uses as its symbol the image of a Kipukamayoc and the Public Accontants do the same, although its not officially the investigated problem is was there a Kipukamayoc in the Wanka Culture along the colonization proces in Peru?. Who was the last Kipukamayoc in the new historial back ground of the Public Accountants and economits in the Wanka culture and ethnics, the quipu and Kipucamayoc and its usage in the conquest and colonization time. The achievemcnt of the investigation has a regional and institutional level using a descriptive method and as example, in the investigation $50 \%$ of the ordinary professors from the accounting and economist faculty. The term of studies of this investigation was for a year then. Important Conclusions: The Wanka leaders learned to use the quipu they leagued to the conquerors giving them warriors leaders, animal goods uoches, tools, utensils, etc. Thes were registered in the quipus in every Quipucamayoc. The Incas divided the Wankas in three huamonis: Hatun Xausa, Ananhuanca and Junin huanca and when the spaniards
\end{abstract}

\footnotetext{
* Este trabajo de investigación fue recibido el 20/12/2005 retornado para su revisión 20/06/2007 y aprobado para su publicación 10/12/2007

1. maguero@hotmail.com

2.silviamarinaa@hotmail.com
} 
got to Cajamarca these were governed bye Ap Manco Surichaqui, Apo Alaya chuquillanqui and Apo Manco Guacrapgucar as the last Quipucamayoc of the Wanka Culture of Wanka ethnia, being baptized the spaniards as Jeronimo Guacrapgucar who registered in the quipus the services and devoted goods quontily giver to the spanish people for the conquest and colonization of Peru. Besides, theres some similarity in the Public Accountants and Economist work with the Quipucamayoc when they existed.

Keys words: statistican, ccountable, Huamanis, quipu, yupana

\section{INTRODUCCIÓN}

El problema investigado fue: ¿existió el Quipucamayoc en la cultura Wanka en el proceso de colonización del Perú? ¿Quién es el último Quipucamayoc de la cultura Wanka? y el objeto de estudio conocer los antecedentes históricos de los Contadores Públicos y de los Economistas, para ampliar los conocimientos hacia la cultura de la doctrinas contables yeconómicas de nuestra región.

Los objetivos genéricos planteados fueron: conocer los antecedentes históricos post inca de los Contadores Públicos y Economistas en la cultura Wanka, como específicos: 1. Conocer los antecedentes de las profesiones de ciencia económica en el Perú y en la UNCP y 2. Especificar las responsabilidades asumidas por el Quipucamayoc en el periodo de la conquista y colonización. El mayor objetivo logrado ha sido incrementar los conocimientos de los antecedentes de los profesionales de contabilidad y economía en la historia pre inca e inca, lográndose identificar al último Quipucamayoc de la cultura Wanka.

El alcance de la presente investigación fue regional e institucional pues involucra a los contadores públicos y economistas de la Región Junín, especialmente a los docentes y estudiantes de las facultades de Contabilidad y de Economía de la Universidad Nacional del Centro del Perú El contenido del presente trabajo, enriquece los conceptos básicos de los hechos y sucesos acontecidos a fines del reino inca y principio de la conquista y colonización del Perú. Al final, se presenta conclusiones, recomendaciones y las referencias bibliográficas utilizadas en el presente caso.

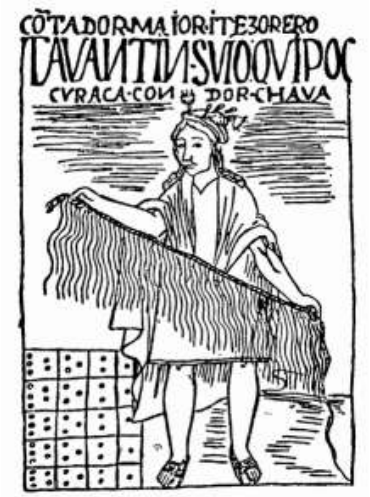

\section{MATERIAL Y MÉTODOS}

\section{Material}

Siendo una investigación descriptiva, se ha utilizado fuentes bibliográficas publicadas, como textos, revistas, periódicos y otras publicaciones. También se ha utilizado información de internet.

Método.- El método que se ha utilizado en la presente investigación es el descriptivo con entrevistas a los profesores de las Facultades de Contabilidad y de Economía.

Determinación de la población y muestra.- Por ser un universo muy pequeño, se ha tomado en forma directa el número de la muestra.

\section{a) Población y muestra}

Población: 100\% del personal docente nombrado y con asistencia normal de las facultades de Economía y de Contabilidad registrada al mes de julio 2005.

$\begin{array}{ll}\text { Facultad de Economía } & 22 \\ \text { Facultad de Contabilidad } & 22 \\ \text { Total población } & 44\end{array}$

Muestra: 50\% del personal docente nombrado de las Facultades de Economía y de Contabilidad.

$\begin{array}{ll}\text { Facultad de Economía } & 11 \\ \text { Facultad de Contabilidad } & 11 \\ \text { Total muestra } & 22\end{array}$

b) Técnicas de procesamiento y análisis de datos.- La muestra no fue seleccionada sino, se aplicó el criterio al azar con el requisito de ser docente ordinario, sin tener en cuenta la categoría, sexo, edad, adscripción al Departamento Académico, tiempo de servicios. Al docente se le pregunta: ¿los incas introdujeron el manejo de los quipus en todas las etnias conquistadas, sabe o conoce Ud. a la llegada de los españoles, quién fue el último Quipucamayoc de la cultura Wanka? Los docentes entrevistados, no indicaron nombre alguno en formato preelaborado. 


\section{RESULTADOS}

\section{De las profesiones del contador y del economista}

Antecedentes históricos de la Contabilidad y Economía.- La contabilidad se inicia con "El fraile franciscano Luca Pacioli (1450-1520), eminente matemático de Milán, quien propone un sistema de doble registro basado en la ecuación de los bienes cuantificados en moneda, forman con los derechos, también cuantificados sobre esos bienes, es decir, Activo = Participaciones. Pacioli, amigo y maestro de Leonardo, viajó con él por la Europa Central dictando conferencias. Su tratado "La divina proporción", fue ilustrado por Leonardo y en él describe los teoremas geométricos que le dieron tanto prestigio, sin embargo, es por la intervención del sistema de Contabilidad llamado de "partida doble" por lo que es más conocido" (Balwind, 1985). A partir de Pacioli, la contabilidad desarrolla el sistema que permite registrar operaciones que pueda cuantificarse en dinero, anotando los bienes al lado izquierdo y las participaciones al derecho.

Época incaica.- Castañeda Quispe (1988) señala "...los incas tuvieron, al igual que los maya o hindũes, un sistema de numeración basado en el valor de la posición de los signos, los cuales, en vez de ser gráficos, tomaban la forma de nudos situados a lo largo de una cuerda y eran llamados quipus (término quecha: quipuni). La numeración incaica, por ser decimal, se identificaba más que con la de los mayas, con la numeración de la India y presentaba, consecuentemente, gran parecido con el sistema que practicamos en la actualidad mediante el empleo de los denominados números arábigos. Esto significa que con los quipus se podían realizar perfectamente las operaciones de cómputo, sin tener que recurrir, forzosamente, al empleo del ábaco, como tuvieron que hacerlos aquellos pueblos que desconocieron el valor de la posición de las cifras" (Castañeda, 1988).

Instrumento del Quipucamayoc: la yupana.- El mismo autor indica "La yupana es el ábaco que utilizaron los Quipucamayocs o contadores del imperio incaico. El vocablo yupana deriva de la palabra quechua "yupay" que significa contar" (Castañeda, 1988). El autor citando a William Burns, indica que cada una de las columnas, de derecha a izquierda, representa un orden numérico en el sistema de numeración decimal: unidades primer orden, decenas, centenas; unidades de millar y decenas de millar, respectivamente.

\section{EL CONTADOR EN LA CONQUISTA Y LA COLONIA}

El Rey de España, el 15 de mayo de 1529, nombra a don Antonio Navarro para que sea el Contador de las nuevas tierras en la que se incluye el Imperio del Tahuantinsuyo. El
24 de agosto de 1605, el Rey de España crea el Tribunal de Contaduría Mayor de Cuentas, y en Lima en febrero de 1607, para controlar las fuentes de ingresos de la Hacienda Pública constituidos por los quintos, diezmos, almojarifazgos, venta de empleos y títulos, impuestos de avería y de alcabala.

\section{EL CONTADOREN LAREPÚBLICA}

Después de la Independencia del Perú, se promulga la Constitución de 1823, que en su artículo 153 establece "Habrá también en la Capital de la República una Tesorería General compuesta por un Contador, un Tesorero y de los empleados correspondientes; se reunirá en ella todos los productos de la Hacienda" (Castañeda, 1988). El 11 de setiembre de 1959, se expide la Ley de Profesionalización del Contador Público No 13253. El 4 de junio 1987 se promulga la Ley $N^{0} 24680$ del Sistema Nacional de Contabilidad.

Antecedentes históricos de la Facultad de Ciencias Económicas.- Con el nombre de Facultad de Ciencias Políticas y Administrativas se crea el 7 de abril de 1875, en la Universidad Nacional Mayor de San Marcos; con Ley $N^{\circ}$ 6041 del 19 de mayo de 1928, se crea la facultad de Ciencias Económicas y a partir del 1935 se expide los grados y títulos siguientes: Bachiller en Ciencias Económicas, Doctor en Economía, Bachiller en Comercio, Contador Público, entre otras especialidades.

La Facultad de Ciencias Económicas pasó a tener la denominación de Facultad de Ciencias Económicas y Comerciales y en 1950 se incorpora la especialidad de Administración, con lo cual se inicia la expedición de títulos de: Economista, Contador Público, Administrador.

La creación de las Facultades de Economía y Contabilidad en la UNCP.- Con el D. S. N ${ }^{0} 046$ del 16 de diciembre 1959 en su Art.1․ Reconoce la fundación y autoriza el funcionamiento de la Universidad Comunal del Centro del Perú, como privada con sede en la ciudad de Huancayo. Entre ellas se encuentran las: facultad de Economía, Estadística y Finanzas y la Facultad de Contabilidad, Comercio y Cálculo Actuarial

\section{CREACIÓN DE LOS COLEGIOS PROFESIONALES DE CONTADORES PÚBLICOS YDE ECONOMISTAS}

El Colegio de Contadores Públicos del Perú.- En Lima, 17 de julio 1942 los Contadores Públicos, se reunieron para forjar desde el seno institucional la superación profesional de cada uno de sus miembros y decidieron formar el Primer Colegio de Contadores Público. El 26 de agosto de 1942 se instaló la primera Directiva bajo la presidencia del C.P. Marcial García Pantoja, en virtud del Art. 5 de la Ley 13253 y su Reglamento del 26 de agosto de 1960. 
El Colegio de Economistas del Perú.- Como se recuerda con fecha 8 de abril de 1965, Ley del Economista № 15488, día 23 de octubre 1965 en Lima, adjunto el proyecto de Estatuto del Colegio de Economista del Perú, aprobados por la Comisión Nacional, y en relación con la disposición transitoria del mismo, se acuerda constituir el Colegio de Economistas de Lima, en favor de la profesión del Economista.

El Colegio de Contadores Públicos de Junín.-El Colegio de Contadores Públicos de Junín se remonta al año 1966. En el periodo 1966 a 1972, estuvo bajo la dependencia del Colegio de Lima, siendo su Decano el CPC. Vicente Portocarrero Valverde, Director Secretario CPC. Adriel Osorio Zamalloa y como Director Tesorero CPC. Juan Alberto Barandiarán Requejo.

Formada la Comisión Organizadora del Colegio de Contadores Públicos de Junín en el periodo 1978-79 con la presidencia del CPC Félix Ríos Bianchi, el 23 de noviembre de 1978 se institucionaliza como un organismo autónomo.

El Colegio de Economistas de Junín.- El 8 de abril de 1965, se expide la Ley 15488 de la Ley del Economista siendo el primero el Colegio de Economista de Lima. El 14 de octubre de 1978, se crea el Colegio de Economista de Junín, con sede en Huancayo, en base a la Ley 15488. Su primer Decano fue el Economista Juan Privat Gómez (ex alumno de la Facultad de Ciencias Económicas de la UNCP, al amparo de la Ley 15488 y 24531 del 14 de junio 1986 y su Reglamento aprobado por D. S. No 041-87-EF publicado el 01 de marzo de 1987.

\section{ATRIBUCIONES DEL CONTADOR PÚBLICO Y EL ECONOMISTA}

Del Contador Público.-El objetivo principal de su trabajo es el mejoramiento de los servicios contables, teniendo como funciones principales y especificas:

a) Es el ejecutivo principal e indispensable en el desarrollo económico, orgánico y administrativo de la empresa, b) Planifica la estructura que se ha de desarrollar dentro y fuera de la empresa con la finalidad de incrementar el desarrollo y extensión orgánica, producción y comercialización con fines de reducción de los costos, c) Prepara o reestructura el Plan General de Cuentas, d) Prepara la racionalización de los sistemas, e) Dirige y controla los registros contables (Asientos) $y, f$ ) Es el contador responsable de los resultados de las operaciones del capital (patrimonial económico y financiero, balance, liquidaciones, compra ventas, cobros, pagos, etc.)

Del Economista.- Roel Pineda, plantea la labor de los economistas en los temas siguientes: a) Orientación del proceso nacional, b) Búsqueda de soluciones ingeniosas y eficaces para resolver nuestros problemas más acuciantes, c) Apoyar los esfuerzos de la población, d) Estimular el desarrollo de un nacionalismo humanista, e) Propiciar hondos y auténticos cambios estructurales, f) Propiciar un cambio profundo de nuestro sistema educativo.

Bunge, Mario (1988) asegura que "en la vida real los economistas suelen ser, no solo estudiosos de la economía, sino también consultores, planeadores, e incluso administradores o políticos" (Bunge, 1988). En la actualidad las funciones del Economista, se ha diversificado, siendo las principales, entre otros, lo siguiente: Investigación, diseño e implementación de políticas económicas en los gobiernos nacionales, regionales y locales. Análisis y diagnóstico empresarial, consultoría, docencia universitaria, entre otros.

\section{DISCUSIÓN}

\section{¿POR QUÉ LOS ECONOMISTAS ELIGEN EL QUIPUCAMAYOC?}

La Ley N 15488, Ley de Profesionalización y Colegiación del Economista en el Perú fue dada el 8 de abril de 1965. El primer Decano el Econ. Luis Felipe De Las Casas Grieve y su Consejo Directivo buscaron un símbolo que represente lo que significaba la nueva profesión. El Quipucamayoc fue el elegido y en efecto representa el conocimiento de sistemas estadísticos y numéricos, de pronósticos, de administración de recursos humanos, materiales y tributarios, el manejo de censos e inventarios, etc.; en suma era el planeamiento estratégico del sistema socio-económico del Imperio de los Incas. Era la profesión del economista con las limitaciones de la antigüedad, tenía el quipu como herramienta auxiliar a la memoria.

\section{LACULTURA WANKA, EL QUIPUYEL QUIPUCAMAYOC}

La incorporación de la cultura Wanka al dominio de los incas.- Espinoza Soriano (1972) indica que los wankas fueron vencidos por Túpac Yupanqui Inca, por hambre y sed, ya que sus provisiones se agotaron, una vez derrotados, sus defensores fueron deportados en masa a otras regiones, dejando despoblada la capital Siquillapucara. Esto fue dividida en tres sayas: al norte se le dio el nombre de Jatunsausa como cabecera la Llacta del mismo nombre; la segunda ubicada en el centro, llamada Lurinhuanca cuya capital fue la Llacta de Tuna y, la tercera al sur, fue Ananhuanca, cuya capital fue La Llacta de Sicaya, así perdieron el rango de reino y se convirtieron en Wanka huamani o provincia del Tahuantinsuyo.

El Tahuantinsuyo lo integraban más de doscientos reinos, con gente traída o deportada a otras regiones del imperio, ese hecho generó odio hacia los incas y los reinos conquistados socavaron la religión y los fundamentos del 
Estado Inca. Los wankas no son exceptuados de este pensamiento. Espinoza Soriano (1972) señala: "Así, los huancas y españoles pactaron la alianza, porque, a ambos les convenía: el imperio no estaba en condiciones de desplegar toda su capacidad militar, política, económica y espiritual para hacer la guerra al invasor, porque el afán de la independencia no había sido sepultado en los reinos" (Espinoza, 1972), por esta razón la mayoría de los señoríos se plegaron a los españoles, entre ellos los wankas.

Pizarro, como hábil político, trabajó con los sentimientos y resentimientos de los pequeños reinos. Fueron estos pactos, que no emanaron del amor al arte, sino de los intereses particulares de ambas partes, lo que determinaron la caída del Imperio. "en primer lugar los curacas huancas pensaron obtener así la ayuda del español para desgajarse de la monarquía cuzqueña y proclamar nuevamente su gloriosa independencia. Por su parte, los españoles meditaron lograr el auxilio de los huancas en víveres, cargueros, guerreros y mujeres para marchar contra la clase dirigente del Tahuantinsuyo" (Espinoza, 1972), Desde luego que lo hicieron prometiéndoles libertad, así los wankas salieron de un amo para caer en el otro amo.

\section{La incorporación de la cultura Wanka al dominio de los} españoles.-Francisco Pizarro, inicia su proceso de conquista y colonización con la fundación de la Villa de San Miguel de Piura un 15 de junio de 1532. En Cajamarca es apresado y asesinado el Inca Atahualpa el 26 de julio de 1533, Francisco Pizarro llega a la zona habitada por los Xauxas, aquí es recibido por los principales y población en general, Pizarro ve las condiciones inmejorables de la zona y decide contar con una nueva capital para efectos de la conquista, así el 4 de octubre de 1533 funda la ciudad de Jatun Xauxa dejando algunos españoles y el 25 de abril 1534, reitera la fundación de Jauja

Para evitar los abusos y excesos de los españoles con los indígenas, el 3 de julio de 1565 se crea el Corregimiento de Jauja a cargo de Juan Larreinaga Salazar. De esta manera, la capital de los wankas fue incorporada y administrada por los españoles,

Aportes de los wankas para la conquista y colonización del Perú.- Espinoza Soriano (1972) menciona entre la información presentada por los hijos de los Apo Manco Surichaqui, Apo Manco Guacrapaucar y el Apo Alaya Chuquillanqui como son don Francisco Cusichaqui, don Felipe Guacrapaucar y don Carlos Alaya, respectivamente anota los pedidos de recompensa por los servicios prestados a los conquistadores españoles. Es así que el 15 de julio 1558 éstos, presentan ante el Presidente y Oidores de la Audiencia Real las memorias descritas en los quipus, que dice "Memoria de los indios que don Jerónimo Guacrapaucar dio al marqués don Francisco Pizarro desde que salió de Caxamarca. Son los siguientes:" (Espinoza, 1972), Los indios que dio al marqués:

1) Primeramente se perdieron en aquella jornada 596 indios y mujeres 119.

2) Más le dimos en oro y plata en Caxamarca en otros 596 pesos en plata le di nosotros tantos que son 595 pesos....1292

3) Más dimos en ropa de cumbe 80 piezas de ropa de hombres y de mujeres...80

4) Más le dimos cuatro mantas de caballo...4

5) Más le dimos cuarenta carneros ...40

6) Más le dimos 149 hanegas de maiz...149

7) Cuando el marqués fue a Bombón le dimos 826 indios y todos se perdieron en la jornada...826

8) Más le dimos a Soto capitán por mandado del marqués 37 indios y 45 indias y todos estos indios y indias murieron en la batalla que dieron al inga Yucra Guallpa.

... continúan los aportes hasta el 158)

Igualmente en las Memorias: De las cosas que don Cristóbal Alanya cacique e indios dieron a los capitanes y gente de Su Majestad para la guerra contra Francisco Hernández Girón. Memoria de don Cristóbal:

1) 296 marcos de cobre

2) 39 marcos de plomo

3) 3 pares de vestidos para indios

4) Untoldo,

... continúa hasta el número 55)

En la Memoria y relación de lo que yo don Alvaro cacique de los indios de Caravantes gasté con los capitanes de su Majestad en la guerra de Francisco Hernández y diferente della. Otro de donAlvaro.

1) 1986 indios de carga

... continúa hasta el número 46)

Memoria y relación de lo que con los capitanes de Su Majestad gasté yo don Jerónimo en la guerra de Francisco Hernández y diferente della. Otra de don Jerónimo.

1) 3262 indios de carga

2) 2696 fanegas y seis salmudes de maíz.

... continúa hasta el número 44)

Existen informaciones que ofrecen los testigos de los 3 Apus de Jatunxauxa, Lurinhuanca y Ananhuanca para probar los favores entregados a Francisco Pizarro reconociendo a los conquistadores como los salvadores que habían apresado y asesinado al Inca Atahuallpa. Toda esta información de los hombres, mujeres, bienes entregados y servicios prestados a los invasores en el imperio incaico fueron registrados en los quipus. 
El Quipu.- El Quipu ("nudo" o "cuenta por nudos"): es un instrumento memotécnico empleado por los Incas para llevar la cuenta de los tributos, la estadística demográfica, el recuerdo de sus hechos pasados, así como la razón de sus leyes y ordenanzas, ritos y ceremonias. Según la descripción de Garcilaso, que manejó los quipus llevados por los indios pertenecientes a la encomienda de su padre, cuando acudían al Cusco para pagar sus tributos: "Hacían los indios hilos de diversos colores: unos eran de un color solo, otros de dos colores, otros de tres y otros de más, porque los colores simples y los mezclados, todos tenían su significación de por sí: los hilos eran muy torcidos, de tres o cuatro liñuelos y gruesos como un huso de hierro y largos de a tres cuartas de vara, los cuales ensartaban en otro hilo por su orden a la larga, a manera de rapacejos.

Por los colores del hilo, como el oro por el amarillo y la plata por el blanco, y por el colorado la gente de guerra. Las cosas que no tenían colores iban puestas por su orden, empezando de las de más calidad y procediendo hasta las de menos, cada cosa en su género, como en las mieses y legumbres... Algunos de estos hilos tenían otros hilitos delgados del mismo color, como hijuelas o excepciones de aquellas reglas generales, como digamos en el hilo de los hombres o mujeres de tal edad, que se entendían ser casados, los hilitos significaban el número de los viudos 0 viudas que de aquella edad había aquel año, porque estas cuentas eran anuales y no daban razón más que de un año solo. Los nudos se daban por su orden de unidad, decena, centena, millar, decena de millar y pocas veces o nunca pasaban a la centena de millar: porque, como cada pueblo tenía su cuenta de por sí y cada metrópoli la de su distrito, nunca llegaba al número de estos o de aquellos a tanta cantidad que pasase la centena de millar, que en los números que hay de allí abajo tenían harto. Hubo depósitos de quipus en Cusco.

Castañeda (1988) señala que "el quipu en sí dice Cieza "ramal grande de cuerdas anudadas" consistía en una cuerda gruesa y horizontal de la que hacían pender cantidad de cuerdas delgadas y verticales; estas últimas lucían nudos de diferentes clases y colores e igualmente algunas cuerdecillas subsidiarias. El material de todas estas cuerdas fue el algodón insertándose raramente en cu confección mechones de lana y hebras de cabellos humanos. Los quipus eran de diferente tamaño, existiendo cuerdas gruesas horizontales hasta $2.65 \mathrm{~m}$ de longitud y verticales, de hasta $67 \mathrm{~cm}$ de largo".

El Quipucamayoc.- El Quipucamayoc, educado por los amautas en escuelas especiales llamadas Yachayhuasi, era el especialista en elaborar, "leer" y archivar los quipus, podía ser de la nobleza, de no serlo era un "honorable" dotado de una memoria prodigiosa. "Honorables" como grupo social eran los especialistas calificados. Algunos autores, creen ver en ellos un "embrión de la clase media".
Apoyaban a los administradores de los collcas o depósitos; a los agrimensores para distribuir la tierra; a los cobradores de impuestos, para el control de los contribuyentes y los ingresos fiscales; a los astrólogos para predecir la época de la siembray de la cosecha.

Puede decirse que el Quipucamayoc era lo que es hoy el analista económico 0 el responsable del planeamiento estratégico, igualmente el quipu para los incas, era lo que es hoy el moderno computador para los economistas.

El Quipucamayoc o Contador era un funcionario que por sus conocimientos alcanzaba la más alta jerarquía entre los oficios existentes, por lo que sus funciones eran: a) Elaborar y hacer las anotaciones en los quipus, b) Consolidar la información de la contabilidad en base a los datos (quipus) proporcionados por los contadores regionales. Para ello, su organización contaba con 4 regiones (suyo) y, c) Archivar los quipus. El Quipocamayoc era el encargado de guardar los quipos, daba cuenta de las disposiciones y ordenanzas, ritos y ceremonias, actos éstos que se identificaban por el color de los hilos, así como por la variedad de sus nudos.

\section{ELÚLTIMO QUIPUCAMAYOC DE LA CULTURA WANKA}

En 1532, el Apo Manco Surichaqui fue el curaca de la saya de Jatunsausa bautizado en 1534 como Francisco que fue conocido con el apelativo de Francisco Xauxa Cusicha. En saya de Lurinhuanca tiene al jatuncuraca Apo Manco Guacrapaucar, vivía en la Llacta de Tunan. En la saya de Ananhuanca en 1532 era el curaca Apo Alaya vivía en la Llacta de Sicaya. Estos y otros reinos desconformes con los incas, acogieron a Pizarro, éste lo tomó como un milagro que debía aprovecharlos desde el punto de vista político.

Los mismos curacas, entregaron mayor número de guerreros, ropas, alimentos, objetos de oro y plata solicitando que eliminara a los ejércitos del inca. Cuando Cieza de León, quien deseaba conocer el manejo de los quipus solicito a Guacrapaucar, en Maravilca le mostró los cordeles anudados en que constaba la relación completa de los productos que habían dado a Pizarro desde 1532 citado por Espinoza Soriano: "yo estaba incrédulo de esta cuenta, y aunque lo oí afirmar y tratar, tenía lo más bello por fábula; $y$ estando en la provincia de Xauxa, en lo que llaman Maravillca, rogué al señor Guacarapora (sic) que me hiciese entender la cuenta dicha de tal manera que yo me satisficiese a mi mismo, para estar cierto que era fiel y verdadera. Y luego mandó a sus criados que fuesen por los quipos, y como este sea de buen entendimiento y razón por ser indio, con mucho reposo satisfizo a mi demanda. Y me dijo, que para que mejor lo entendiese, que notase que todo lo que por su parte había dado a los españoles desde que entró el gobernador don Francisco Pizarro en el valle, estaba allí sin faltar nada. Y así vi la cuenta del oro, plata, ropa que habían dado, con todo el maíz, ganado y otras 
cosas, que en verdad yo quedé espantado dello" (Espinoza, 1972),.

Así la alianza entre wankas y españoles funcionó a plenitud, porque desde el valle de Jatunmayo, partían las órdenes para aplacar las rebeliones o conquistas. En 1558 Francisco Guacrapaucar, don Francisco Cusichaca y don Carlos Apo Alaya, principales de la nación Wanka, dictaron a un escribano unas memorias sobre la colaboración de ellos en el apaciguamiento de Francisco Hernández Girón, con el justo pago de todos los gastos hechos en ella, estas memorias fueron remitidos al Consejo de Indias en consulta.

Los 3 curacas viajaron a Lima con testigos llevando información más detallada que las simples memorias de 1558 para exponer de todos los servicios y la integridad de la ayuda que los Wankas brindaron a los españoles desde 1532 hasta 1560 y 1561 . Cada uno hizo un informe sobre la alianza con los españoles: a) de Jatunxauxa representado por Francisco Cusichaqui o Cusichac (Jauja), b) de Lurinhuanca representado por Felipe Guacrapaucar (Tuna o Tunán) y, c) de Anahuanca representado por Carlos Alaya (Sicaya). La documentación fue presentada al Consejo de Indias, el cual se extravió el de Ananhuanca. Felipe Guacrapaucar viajó a España en representación de su padre (Apo Manco Guacrapaucar) y de Francisco Cusichaqui (hijo de Apo Manco Surichaqui).

Felipe Guacrapaucar llegó a Lima en junio de 1560, el 23 del mismo mes y año, se dirigió a la Audiencia, solicitando a nombre de su anciano padre, realizar ante los oidores una probanza acerca de la actuación del Apo Jerónimo Guacrapaucar y de la saya de Lurinhuanca desde 1533 año en que se aliaron con Francisco Pizarro, portando consigo un manojo de quipus además llevó 17 testigos. El 26 de agosto de 1560, se terminó de escribir y recibir la información de don Jerónimo Guacrapaucar y de la saya de Lurinhuanca, a pedido de Felipe Guacrapaucar, se expedió copia para llevar a España.

\begin{tabular}{lll}
\hline $\begin{array}{c}\text { Contador } \\
\text { Público }\end{array}$ & Quipucamayoc & Economista \\
\hline - Registro & - Elaborar y & - Elaboración \\
contable y & hacer las & estadísticas \\
teneduría de & anotaciones & - Administración \\
libros & en los quipus. & de recursos \\
- Administración & - Consolidar la & - Elaborar \\
de recursos & información & inventarios \\
- Base de datos & de la & - Base de datos \\
- Elaborar & contabilidad & - Elaboración \\
inventarios y & en base a los & de \\
balances & datos (quipus) & diagnósticos \\
- Conservación & proporcionados & - Elaborar \\
de información & por los & memorias \\
y & contadores & \\
documentación & regionales & \\
& - Archivar los & \\
& quipus & \\
& &
\end{tabular}

Se aprecia que existen funciones similares al rol que desempeñan en parte el Contador Público y el Economista en la actualidad.

\section{CONCLUSIONES}

- Al ser conquistados por los Incas, los líderes wankas, aprendieron a utilizar el quipu, siendo así que a la llegada de los españoles al Valle del Huancamayo, se aliaron a los conquistadores entregándoles guerreros, cargadores, animales, bienes como alimentos, vestidos, herramientas, enseres, utensilios, etc. Ios mismo que fueron registrados en el quipu de cada Quipucamayoc, por lo que existió y se utilizaron los quipus en 1533

- El Quipucamayoc ha utilizado un instrumento de cálculo denominado YUPANA que sirvió para tener una cantidad total para luego ser anotado en el quipu.

- Los incas dividieron a los wankas en 3 huamanis: Jatun Xausa, Anan Wanka y Lurin Wanka y a la llegada de los españoles a Caxamarca, estos huamanis estaban gobernados por: Apo Manco Suchaqui, Apo Alaya Chuquillanqui y, Apo Manco Guacrapaucar, respectivamente, quienes conocían el manejo de los quipus, considerándose al Apo Manco Guacrapaucar como el último Quipucamayoc de la cultura o etnia Wanka que tomó el nombre de Jerónimo Guacrapaucar, que tuvo una edad mayor a los otros y quien ha registrado en los quipos la cantidades de servicios y bienes entregados a los españoles para la conquista y colonización del Perú

- Existen funciones que fueron ejecutadas por el Quipucamayoc que son similares a las funciones en la actualidad del Contador Público y del Economista.

\section{LITERATURA CITADA}

Baldwin, J. G.1985. Manual de Contabilidad. Tomo I Editorial del Castillo S.R.LLima. Pág. 15

Bunge, M. 1988. Economía y Filosofía, Editorial Ciencias Sociales La Habana. Pág. 63

Castañeda Quispe, A. 1988. La Contabilidad para el desarrollo. Editorial Perugraph Editores. Lima. Pág. 39

Espinoza Soriano, W. 1972. Los Huancas, aliados de la conquista, publicado en Anales Científicos de la Universidad del Centro. Pág. 59. 